\title{
Natural orifice surgery: initial clinical experience
}

\author{
Santiago Horgan · John P. Cullen · Mark A. Talamini · Yoav Mintz · \\ Alberto Ferreres · Garth R. Jacobsen · Bryan Sandler · Julie Bosia • \\ Thomas Savides · David W. Easter · Michelle K. Savu · Sonia L. Ramamoorthy · \\ Emily Whitcomb - Sanjay Agarwal - Emily Lukacz · Guillermo Dominguez · \\ Pedro Ferraina
}

Received: 11 September 2008/Accepted: 11 February 2009/Published online: 3 April 2009

(C) The Author(s) 2009. This article is published with open access at Springerlink.com

\begin{abstract}
Background Natural orifice translumenal endoscopic surgery (NOTES) has moved quickly from preclinical investigation to clinical implementation. However, several major technical problems limit clinical NOTES including safe access, retraction and dissection of the gallbladder, and clipping of key structures. This study aimed to identify challenges and develop solutions for NOTES during the initial clinical experience.

Methods Under an Institutional Review Board (IRB)approved protocol, patients consented to a natural orifice operation for removal of either the gallbladder or the appendix via either the vagina or the stomach using a single umbilical trocar for safety and assistance.

Results Nine transvaginal cholecystectomies, one transgastric appendectomy, and one transvaginal appendectomy have been completed to date. All but one patient were discharged on postoperative day 1 as per protocol. No complications occurred.
\end{abstract}

S. Horgan · J. P. Cullen ( $)$ - M. A. Talamini · Y. Mintz ·

G. R. Jacobsen - B. Sandler · J. Bosia - D. W. Easter ·

M. K. Savu · S. L. Ramamoorthy

Department of Surgery, University of California San Diego, 200 W. Arbor Drive, 8402, San Diego, CA 92103, USA

e-mail: jcullen@ucsd.edu

A. Ferreres · G. Dominguez · P. Ferraina

Department of General Surgery, Buenos Aires Hospital de

Clinicas, Buenos Aires, Argentina

T. Savides

Department of Gastroenterology, University of California

San Diego, San Diego, CA, USA

E. Whitcomb · S. Agarwal - E. Lukacz

Department of Reproductive Medicine, University of California

San Diego, San Diego, CA, USA
Conclusion The limited initial evidence from this study demonstrates that NOTES is feasible and safe. The addition of an umbilical trocar is a bridge allowing safe performance of NOTES procedures until better instruments become available. The addition of a flexible long grasper through the vagina and a flexible operating platform through the stomach has enabled the performance of NOTES in a safe and easily reproducible manner. The use of a uterine manipulator has facilitated visualization of the cul de sac in women with a uterus to allow for safe transvaginal access.

Keywords Appendix - Cholecystectomy ·

Clinical papers, trials, research $\cdot$ Endoscopy $\cdot$ Surgical

Natural orifice translumenal endoscopic surgery (NOTES) is a novel surgical technique that may improve patient outcomes in minimally invasive surgery. The clearest benefit is cosmetic because surgeons use the body's natural orifices for access rather than transfascial incisions. Leaders in gastroenterology and surgery anticipate that NOTES will reduce the incidence of hernia and may improve pain and recovery [1].

In much the same way as laparoscopy 20 years ago, NOTES defies conventional surgical practices and has been the subject of some appropriate skepticism. In 2004, Kalloo et al. [2] described NOTES in an animal model as a potential next step in the evolution of therapeutic endoscopy. Although several variations of natural orifice operations predate the work of Kalloo et al. [2], it was their first experiments that sparked the current heavy interest in NOTES.

In response to the clinical potential of NOTES, leaders from the pertinent surgery society (Society of American 
Gastrointestinal and Endoscopic Surgeons [SAGES]) and American Society for Gastrointestinal Endoscopy (ASGE) generated a white paper that encouraged further NOTES research and outlined key research areas that needed to be addressed [3]. This outline, generously funded, has led to a body of preclinical work over the past few years. Notably, leaders agreed that all NOTES cases be collated in a central database to ensure accurate reporting of outcomes and to provide early evidence of important trends regarding the safety of NOTES.

A large body of preclinical evidence now exists, demonstrating that several types of NOTES operations can be performed in both acute and survival animal models through a variety of approaches including access via the stomach, rectum, vagina, esophagus, and bladder. The NOTES procedure has moved quickly from a concept to preclinical studies to human clinical trials based on this preclinical work.

To date, most of the published clinical series report experience with the transgastric approach. Endoscopic surgery can be used successfully to treat pancreatic pseudocysts [4], and small published trials have demonstrated the use of NOTES in peritoneoscopy [5, 6]. Although several institutions are performing clinical research with hybrid NOTES techniques that have been reported in the lay press, few peer-reviewed articles exist at this writing. The published series to date have been encouraging, including series of transvaginal cholecystectomies [7, 8] and transvaginal appendectomies [9].

At our institution, we invested nearly 2 years in preclinical research using both animate and anatomic material models to maximize patient safety and prepare the way for a clinical trial. Evidence from this intensive work was central to the successful application to the IRB. We described a dual-view hybrid NOTES technique previously, which we believe maximizes safety [10]. The vagina was selected initially as the safest access point for NOTES. Close consultation with the University's Reproductive Medicine and Gastroenterology Departments was central to our planning of human NOTES operations.

This report aims to describe our early clinical experience with transvaginal and transgastric NOTES surgery.

\section{Methods}

Two separate protocols for performing NOTES operations were approved by the University of California San Diego (UCSD) IRB. The transvaginal and transgastric approaches were separated into different protocols. Exclusion criteria for both protocols specified prior open abdominal operations, morbid obesity, and extremes of age (Table 1). presents a full list of exclusion criteria obtained by the principal investigator in all cases.
Table 1 Exclusion criteria

1. Patients with a body mass index (BMI) greater than 35

2. Patients younger than 18 or older than 65

3. Patients with a history of severe endometriosis, pelvic inflammatory disease, or ectopic pregnancy

4. Patients with known common duct stones or presumed gallbladder mass

5. Patients with prior open abdominal operations

6. Pregnant women

7. Patients with severe medical comorbidities

8. Patients with a history of vaginal trauma

9. Patients with clotting or bleeding disorders and patients receiving anticoagulant or antiplatelet medications

The protocols were translated into Spanish and submitted to the IRB for Hospital De Clinicas, Buenos Aires, Argentina. The IRB-approved protocol at that institution was identical to that of UCSD. Of our 11 cases, 4 were managed in Argentina. All these cases were transvaginal cholecystectomies.

The management of all cases begins with placement of a small ( $5 \mathrm{~mm}$ or smaller) umbilical trocar for surveillance of the abdomen to determine the feasibility of a natural orifice procedure. All patients are kept in the hospital overnight. The clinical protocol follow-up evaluation includes daily phone calls from the surgeon to check the temperature and pain levels postoperatively and clinic visits at 1 week and 1 month. No additional laboratory or radiologic testing is performed.

\section{Results}

A total of 11 NOTES operations have been performed to date over a 6-month period beginning August 2007. The first five cases were managed in Argentina and the remainder in the United States. Three additional patients consented to NOTES operations, which involved two transvaginal cholecystectomies and one transgastric appendectomy. However, after the initial umbilical trocar was placed, the decision was made not to proceed with a natural orifice approach due to severe inflammation in two cases and adhesions in the third case.

All the patients were discharged on postoperative days 1 and 2. No patient exceeded this hospital stay. The initial patient stayed two nights for social reasons (no ride available). All vaginal colpotomies were performed with the assistance of a gynecologist, and all transgastric procedures were performed with the assistance of gastroenterologists.

None of the patients except one transvaginal patient required narcotic pain medications after discharge. No 
Table 2 Data for the first 11 clinical cases

\begin{tabular}{|c|c|c|c|c|c|c|}
\hline Patient & Operation & Age (years) & Time (min) & Abdominal trocars & Hospital stay & Complications \\
\hline 1 & Transvaginal cholecystectomy & 19 & 150 & 2 & 1 Nights & None \\
\hline 2 & Transvaginal cholecystectomy & 22 & 96 & 2 & $1 \mathrm{Night}$ & None \\
\hline 3 & Transvaginal cholecystectomy & 26 & 70 & 1 & 1 Night & None \\
\hline 4 & Transvaginal cholecystectomy & 39 & 92 & 1 & 1 Night & None \\
\hline 5 & Transvaginal cholecystectomy & 49 & 93 & 1 & 1 Night & None \\
\hline 6 & Transvaginal cholecystectomy & 42 & 114 & 1 & $1 \mathrm{Night}$ & None \\
\hline 7 & Transvaginal cholecystectomy & 35 & 165 & 1 & $1 \mathrm{Night}$ & None \\
\hline 8 & Transvaginal cholecystectomy & 33 & 140 & 1 & $1 \mathrm{Night}$ & None \\
\hline 9 & Transvaginal cholecystectomy & 47 & 110 & 1 & $1 \mathrm{Night}$ & None \\
\hline 10 & Transgastric appendectomy & 42 & 150 & $\begin{array}{l}\text { One plus two } \\
2 \text {-mm ports }\end{array}$ & $1 \mathrm{Night}$ & None \\
\hline 11 & Transvaginal appendectomy & 24 & 78 & 1 & 1 Night & None \\
\hline
\end{tabular}

complications had occurred at the 1-month follow-up visit. The data are described in Table 2 . The operative times were measured from the time of the incision to the time of the closure.

\section{Transvaginal cholecystectomy}

To date, nine patients have undergone transvaginal cholecystectomy. In two of these cases, an additional transabdominal port $(3 \mathrm{~mm})$ was used as a safety precaution. In all the other cases, only a single 5-mm umbilical port was used. The vagina was closed primarily with a suture under direct vision in all cases.

The average patient age was 34 years, and the indications for the operation were gallstone disease. The average operating time was $114 \mathrm{~min}$, and the average blood loss was $20 \mathrm{ml}$.

All but one patient reported no use of narcotic pain medications after hospital discharge. The one patient who required oral narcotics used them 2 days for shoulder pain.

At the follow-up visit, no complications were reported by any of the patients. The patients were advised to observe pelvic rest for 4 weeks postoperatively. All sexually active patients reported a return to normal sexual activity.

\section{Transgastric appendectomy}

In the transgastric cases, one transgastric appendectomy was performed. The patient was a 42-year-old man with $16 \mathrm{~h}$ of abdominal pain, an elevated white blood count, and physical exam and computed tomography (CT) findings consistent with appendicitis.

Because this was the first case of transgastric appendectomy at our institution, two needlescopic instruments were used to assist in gastric closure. At the beginning of the case, G-prox sutures (USGI, San Clemente, CA, USA) were placed to assist with closure. These sutures were secured after removal of the specimen. Because this was the first transgastric case managed, the decision was made to aid the G-prox plication sutures with a single stapler load from a linear laparoscopic stapler. The umbilical port was dilated to allow for the stapler. The operative time was $150 \mathrm{~min}$.

\section{Transvaginal appendectomy}

One case of transvaginal appendectomy has been managed. The patient was a 24-year-old woman with $24 \mathrm{~h}$ of abdominal pain, an elevated white blood count, and CT scan findings consistent with acute appendicitis. The estimated blood loss was minimal, and the operative time was $78 \mathrm{~min}$.

\section{Discussion}

We report what we believe is one of the largest, broadest natural orifice series in the Americas. At the time of this report, 11 patients have undergone natural orifice operations at our institution. Our initial experience demonstrates that NOTES is both safe and feasible for cholecystectomy and appendectomy.

The hybrid NOTES approach

A key risk to placement of a vaginal trocar is the possibility of unrecognized injury to nearby structures, particularly the rectum and sigmoid colon. Currently, laparoscopic vision is the best way to visualize the pelvis directly to ensure that no injury occurs during transvaginal access.

Endoscopic insufflation may be used to maintain pneumoperitoneum, but this approach is more difficult to manage and measure than a standard laparoscopic port approach, which is specifically designed for intraabdominal insufflation. A wider variation in pressure is observed than 
with laparoscopic insufflation [11]. The laparoscopic port and insufflation system ensures that any excess insufflation is noted and quickly addressed. The port also allows passage of a single laparoscopic instrument into the abdomen for use. Until better instruments are developed, having one port available for use with well-developed minimally invasive instruments is important for safe natural orifice surgery at this stage.

Dissection of the gallbladder from the liver bed is difficult using the endoscopic needlehook or the L-shape hook device (Olympus America, Center Valley, PA, USA). Although it is possible to dissect the gallbladder from the liver bed using this hook, its small size makes dissection cumbersome and lengthy. The use of a laparoscopic hook from the umbilical port, at least for the difficult portions of the dissection, allows for a safer and quicker procedure compared with the endoscopic counterparts.

Laparoscopic clips are absolutely necessary for patient safety. Endoscopic clips are not entirely occlusive and not designed to secure the cystic duct. The development of new endoscopic instruments may improve upon current instrumentation, but until then, we believe the safest course is to use a single laparoscopic access umbilical port.

In this initial experience, three patients who consented to the research protocol were withdrawn after initial inspection with the laparoscope. In the first planned transgastric appendectomy, severe inflammation was noted and identification of the appendix, ileum, and cecum was difficult. The decision was made to perform a laparoscopic appendectomy.

In one of the planned transvaginal cholecystectomies, the patient had a history of hysterectomy. At placement of the initial trocar, dense pelvic adhesions were noted, and the transvaginal route was abandoned. In another planned transvaginal cholecystectomy, severe inflammation and scarring in the right upper quadrant were seen. This case was converted to laparoscopic cholecystectomy, but laparoscopic removal proved impossible as well.

As experience with NOTES grows, surgeons may become more comfortable with challenging cases. However, during this initial evaluation phase, the most challenging cases should be managed with laparoscopy or open surgery. Further experience will help delineate specific criteria, but to date, these decisions have been made by the judgment of the operative team.

\section{Novel instruments}

Endoscopes were designed to allow visual inspection of the visceral lumens. Although they may be used to resect small polyps or cauterize small bleeding vessels, the platform simply is incapable of completing larger intraabdominal operations in a quick and reproducible fashion.
Natural orifice surgery requires more capability than current endoscopes offer. Retraction of the gallbladder is difficult with flimsy endoscopic graspers that do not maintain a secure purchase on the gallbladder wall. Furthermore, dedicating one channel of an endoscope to retraction limits vision and prohibits use of that channel by a tissue manipulation instrument through a second channel.

A long articulating grasper placed adjacent to the endoscope through a common port (developed by UCSD and Novare Endosurgical, Cupertino, CA) allows for strong and flexible retraction independent of and offset from the endoscopic platform. This instrument, approximately $75 \mathrm{~cm}$ long, features a cable system that allows for flexibility at the tip and extra degrees of freedom, similar to the wrist of the da Vinci robotic surgical system (Intuitive Surgical, Sunnyvale, CA, USA). This device provides enough rigidity for strength of retraction, but with some flexibility to optimize exposure, and the strength of its grip on the gallbladder wall vastly exceeds that of an endoscopic grasper.

A stable operating platform for the transgastric approach also is a valuable addition. The transport system (USGI, San Clemente, CA) allows passage of additional largercaliber endoscopic instruments. Most importantly, it can be locked into a fixed form to allow the endoscopic operator additional freedom. This device also allows for passage of specialized sutures that cannot be used reliably with standard endoscopes.

The transvaginal approach

Compared with other natural orifice access routes, the transvaginal approach to NOTES imparts the least amount of risk to the patient. However, this approach is controversial given its sensitive and private nature. Numerous vaginal and transvaginal gynecologic procedures are performed daily across the United States. Furthermore, data have shown this approach to be safe and effective. It contrasts heavily with the transgastric approach, in which intentional perforation of the gastric wall is a novel concept.

The largest published series of 100 laparoscopic and combined culdoscopic procedures (hybrid NOTES) using multiple transabdominal instruments resulted only in a single uncomplicated postoperative fever [12]. Additionally, series comparing laparoscopically assisted vaginal hysterectomy with laparoscopic hysterectomy have found similar complication rates despite the use of the vaginal conduit [13, 14]. Published studies have demonstrated a higher incidence of certain complications (bladder injury, blood loss greater than 11 , and vaginal hematoma) using a vaginal approach. [15]. It also should be noted that the incision in a NOTES transvaginal procedure and the 
expected blood loss are less than with vaginal hysterectomy. It could be surmised that the addition of laparoscopic vision used in a NOTES approach should reduce the likelihood of bladder injury or excess bleeding.

In recent years, vaginal hysterectomy has gained momentum in many countries as the operation of choice for benign uterine disease requiring an operation [16]. The route of hysterectomy appears to have little effect on postoperative sexual function [17]. Overall pain scores are improved by a vaginal approach compared with abdominal hysterectomy $[18,19]$. This probably is due to avoidance of an incision in the abdominal wall musculature. This benefit hopefully will extend to NOTES.

Clearly, it is critical to discuss all the potential known and unknown risks of a transvaginal NOTES procedure in obtaining informed consent. The risk of infertility after transvaginal NOTES procedures is unknown, but avoidance of bleeding and inflammation to the pelvis should minimize this potential risk. Although the mere suggestion that this procedure could lead to infertility may discourage many proponents of NOTES, the experience of reproductive medicine suggests that the risk is likely to be very small. The transvaginal approach is sometimes used for delivery of therapy to women with refractory infertility [20, 21], and transvaginal procurement of oocytes has been in practice for more than 20 years [22].

A survey conducted at our institution found that approximately $68 \%$ of women $(n=100)$ would be willing to undergo a transvaginal procedure for gallbladder disease if the complication rate were similar (submitted for publication). Though not yet published, this data is included to describe potential patient acceptance of natural orifice procedures. This is consistent with surveys regarding patient attitudes toward NOTES at other institutions [23]. In our initial experience, two patients declined transvaginal cholecystectomy and one declined transgastric appendectomy due to the novel nature of the operation.

In our experience, vaginal access is quickly and easily obtained under direct vision. Because a dilating (nonbladed) trocar is used to stretch the incision, the incision quickly collapses to a smaller diameter after removal of the trocar. The gallbladder is removed easily from the trocar, either with or without an endobag. Using a vaginal speculum, the incision is easily visualized, and a single stitch can be placed to close the incision without difficulty. Larger studies are needed to determine the true safety and efficacy of the transvaginal approach. A national database is the best means for collecting data on the natural orifice experience.

The transgastric approach

The transgastric appendectomy reported herein demonstrates the safety and feasibility of a transgastric approach to appendicitis. The outcome for the reported patient is similar to the outcome expected from a laparoscopic appendectomy. The use of the USGI transport system creates the capability for using additional tools relative to accomplishing the procedure with a standard two-channel therapeutic endoscope.

Reliable and reproducible closure of the gastrotomy remains the largest challenge for the successful development of NOTES. Although many endoscopic suturing methods or devices are in use or development, most are cumbersome and require extensive training and lab time for their effective use.

Dilation of the gastrotomy appears to be preferable to cutting a long gastrotomy because after the endoscope or operating platform is removed, the dilated gastrotomy shrinks down in size as the uncut muscle contracts. Control of both the pnuemoperitoneum and gas volumes inside the stomach are essential to the success of transgastric NOTES.

\section{The future of NOTES}

The NOTES procedure must be safe and the operations easily replicated if the new technique is to become clinically relevant. The described operative approach addresses many of the technical challenges that hinder NOTES and provides solutions for a safe, rapid, and duplicable operation. Laparoscopic assistance allows for safe vision, minimizing the risk for unrecognized injury during access. The 5-mm port also provides control of insufflation, which may be difficult to maintain and measure using an endoscope alone.

In three cases, the initial umbilical port and laparoscope were placed, and the decision was made not to proceed with a NOTES approach. The first case involved a cholecystectomy for a patient who had undergone a prior hysterectomy. Due to pelvic scarring, the vaginal access was not thought to be safe. The second case involved a severely inflamed gallbladder. This case was converted to laparoscopy, but given the severity of the inflammation, the operation could not be completed laparoscopically.

Most importantly, the 5-mm port permits use of the same instruments used in the gold standard for critical portions of the operation. Current endoscopic clips are not indicated for clipping of the cystic duct, so the laparoscopic clips must be used, necessitating a 5-mm abdominal port.

Without a doubt, a cholecystectomy can be completed using current endoscopic instruments, but if this task results in a 4- to 6-h procedure, the patient then is exposed to unnecessary risks. Endoscopic dissection of the gallbladder from the liver bed is a tedious task that requires extensive experience with therapeutic endoscopy. The handles of endoscopes are not ergonomically suitable for such long procedures and may pose a challenge for the 
surgeon. Alternatively, use of the 5-mm hook from an umbilical port is easily accomplished by a general surgeon familiar with laparoscopy and ergonomically beneficial to the surgeon's hands and back.

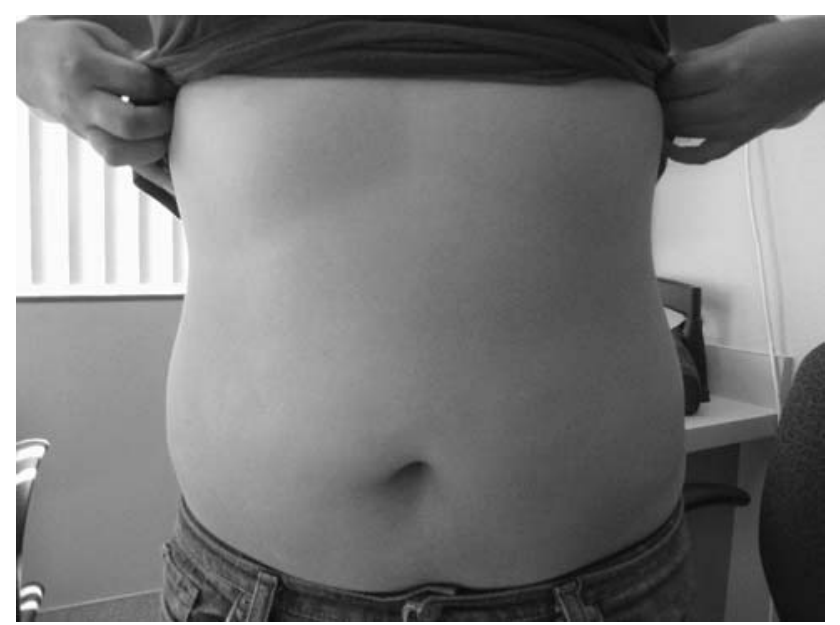

Fig. 1 No visible scar is seen after laparoscopically assisted transvaginal cholecystectomy

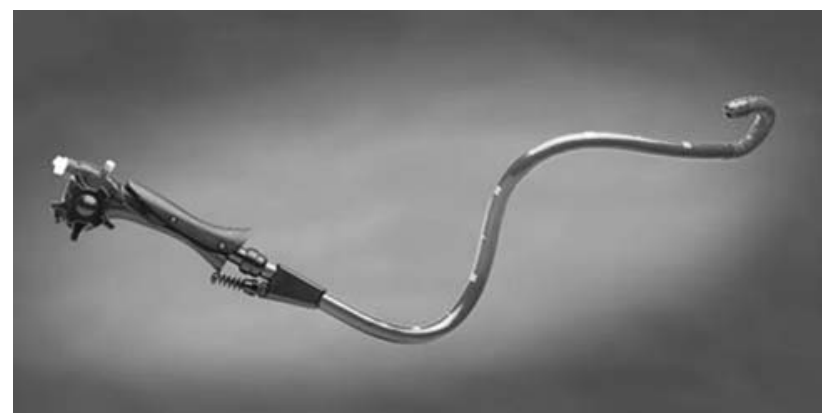

Fig. 2 The transgastric access port from USGI (San Clemente, CA, USA)

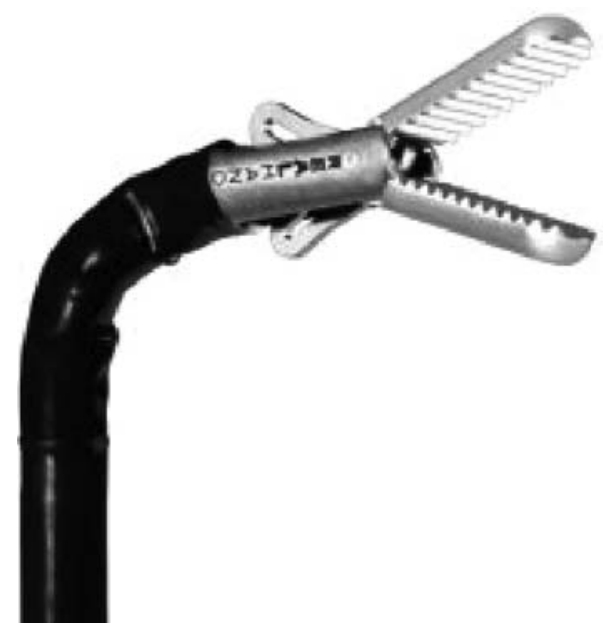

Fig. 3 The flexible tip of the Novare RealHand grasper (Cupertino, CA)
To reduce hernia formation and improve cosmesis, only a single transabdominal trocar is placed. The single $5-\mathrm{mm}$ incision is well hidden within the umbilicus, and the risk of hernia from this single site is low due to the use of a dilating nonbladed trocar. The end effect is a procedure that is virtually scarless (Fig. 1, postoperative view). A pure NOTES approach may eliminate postoperative hernias altogether [1] (Figs. 2, 3).

Further data are needed to determine the true safety and efficacy of clinical NOTES. The creation of the NOSCAR (Natural Orifice Surgery Consortium for Assessment and Research) patient registry ensures an honest review of this emerging technology. With this technique still in its infancy, its potential has created a large interest among researchers and industry to create the next wave of developments in minimally invasive surgery, a rapidly evolving field.

\section{Conclusions}

In this initial series, NOTES proved to be both feasible and safe. Although NOTES introduces a new set of potential complications for natural orifice operations, none were observed in this initial cohort of patients. Patients do not require the oral narcotic medications typically given after these procedures. This may be due in part to sparing of the muscle fibers of the abdominal wall. Placement of the umbilical trocar also spares these fibers and makes use of an existing scar. Still, it is impossible to compare pain outcomes without further prospective study comparing natural orifice surgery with laparoscopic surgery.

Open Access This article is distributed under the terms of the Creative Commons Attribution Noncommercial License which permits any noncommercial use, distribution, and reproduction in any medium, provided the original author(s) and source are credited.

\section{References}

1. McGee MF, Rosen MJ, Marks J, Onders RP, Chak A, Faulx A, Chen VK, Ponsky J (2006) A primer on natural orifice translumenal endoscopic surgery: building a new paradigm. Surg Innov 13:86-93

2. Kalloo AN, Singh VK, Jagannath SB, Niiyama H, Hill SL, Vaughn CA, Magee CA, Kantsevoy SV (2004) Flexible transgastric peritoneoscopy: a novel approach to diagnostic and therapeutic interventions in the peritoneal cavity. Gastrointest Endosc 60:114-117

3. SAGES ASGE (2006) ASGE/SAGES working group on natural orifice translumenal endoscopic surgery white paper October 2005. Gastrointest Endosc 63:199-203

4. Yusuf TE, Baron TH (2006) Endoscopic transmural drainage of pancreatic pseudocysts: results of a national and an international survey of ASGE members. Gastrointest Endosc 63:223-227

5. Hazey JW, Narula VK, Renton DB, Reavis KM, Paul CM, Hinshaw KE, Muscarella P, Ellison EC, Melvin WS (2008) 
Natural-orifice transgastric endoscopic peritoneoscopy in humans: initial clinical trial. Surg Endosc 22(1):16-20

6. Onders RP, McGee MF, Marks J, Chak A, Rosen MJ, Ignagni A, Faulx A, Schomisch S, Ponsky J (2007) Natural orifice transluminal endoscopic surgery (NOTES) as a diagnostic tool in the intensive care unit. Surg Endosc 21:681-683 (Epub 16 February 2007)

7. Zornig C, Mofid H, Emmermann A, Alm M, von Waldenfels HA, Felixmüller C (2008) Scarless cholecystectomy with combined transvaginal and transumbilical approach in a series of 20 patients. Surg Endosc 22:1427-1429 (Epub 9 April 2008)

8. Zorron R, Maggioni LC, Pombo L, Oliveira AL, Carvalho GL, Filgueiras M (2008) NOTES transvaginal cholecystectomy: preliminary clinical application. Surg Endosc 22:542-547 (Epub 20 November 2007)

9. Palanivelu C, Rajan PS, Rangarajan M, Parthasarathi R, Senthilnathan P, Prasad M (2009) Transvaginal endoscopic appendectomy in humans: a unique approach to NOTES: world's first report. Surg Endosc 23(3):668

10. Mintz Y, Horgan S, Cullen J, Ramamoorthy S, Chock A, Savu MK, Easter DW, Talamini MA (2007) NOTES: the hybrid technique. J Laparoendosc Adv Surg Tech A 4:402-406

11. Meireles O, Kantsevoy SV, Kalloo AN, Jagannath SB, Giday SA, Magno P, Shih SP, Hanly EJ, Ko CW, Beitler DM, Marohn MR (2007) Comparison of intraabdominal pressures using the gastroscope and laparoscope for transgastric surgery. Surg Endosc 21:998-1001

12. Tsin DA, Colombero LT, Lambeck J, Manolas P (2007) Minilaparoscopy-assisted natural orifice surgery. JSLS 11:24-29

13. Ghezzi F, Cromi A, Bergamini V, Uccella S, Beretta P, Franchi M, Bolis P (2006) Laparoscopic-assisted vaginal hysterectomy versus total laparoscopic hysterectomy for the management of endometrial cancer: a randomized clinical trial. J Minim Invas Gynecol 13:114-120

14. Muzii L, Basile S, Zupi E, Marconi D, Zullo MA, Manci N, Bellati F, Angioli R, Benedetti Panici P (2007) Laparoscopic- assisted vaginal hysterectomy versus minilaparotomy hysterectomy: a prospective, randomized, multicenter study. J Minim Invasive Gynecol 14:610-615

15. Milad MP, Morrison K, Sokol A, Miller D, Kirkpatrick L (2001) A comparison of laparoscopic supracervical hysterectomy vs laparoscopically assisted vaginal hysterectomy. Surg Endosc 15:286-288 (Epub 12 December 2000)

16. McCracken G, Lefebvre GG (2007) Vaginal hysterectomy: dispelling the myths. J Obstet Gynaecol Can 29:424-428

17. Roussis NP, Waltrous L, Kerr A, Robertazzi R, Cabbad MF (2004) Sexual response in the patient after hysterectomy: total abdominal versus supracervical versus vaginal procedure. Am J Obstet Gynecol 190:1427-1428

18. Morelli M, Caruso M, Noia R, Chiodo D, Cosco C, Lucia E, Biamonte M, Zullo F (2007) Total laparoscopic hysterectomy versus vaginal hysterectomy: a prospective randomized trial. Minerva Ginecol 59:99-105

19. Abdelmonem A, Wilson H, Pasic R (2006) Observational comparison of abdominal, vaginal, and laparoscopic hysterectomy as performed at a university teaching hospital. J Reprod Med 51:945-954

20. Casa A, Sesti F, Marziali M, Gulemì L, Piccione E (2003) Transvaginal hydrolaparoscopic ovarian drilling using bipolar electrosurgery to treat anovulatory women with polycystic ovary syndrome. J Am Assoc Gynecol Laparosc 10:219-222

21. Fernandez H, Alby JD, Gervaise A, de Tayrac R, Frydman R (2001) Operative transvaginal hydrolaparoscopy for treatment of polycystic ovary syndrome: a new minimally invasive surgery. Fertil Steril 75:607-611

22. Schulman JD, Dorfmann AD, Jones SL, Pitt CC, Joyce B, Patton LA (1987) Outpatient in vitro fertilization using transvaginal ultrasound-guided oocyte retrieval. Obstet Gynecol 69:665-668

23. Varadarajulu S, Tamhane A, Drelichman ER (2008) Patient perception of natural orifice transluminal endoscopic surgery as a technique for cholecystectomy. Gastrointest Endosc 67:854-860 (Epub 20 March 2008) 